\title{
An Uncommon Focal Liver Lesion: Intrahepatic Splenosis
}

\author{
Vito Sansone ${ }^{1}$, Lorenzo Falsetti ${ }^{2}$, Francesco Tovoli ${ }^{1}$, Rita Golfieri $^{3}$, Matteo Cescon ${ }^{4}$, Fabio Piscaglia $^{1}$
}

1) Unit of Internal Medicine,

University of Bologna,

Azienda Ospedaliero

Universitaria S.Orsola-

Malpighi, Bologna

2) Internal and Sub-intensive

Medicine Department, A.O.U.

„Ospedali Riuniti”, Ancona

3) Radiology Unit, S.Orsola-

Malpighi Bologna Authority

Hospital, Bologna

4) Department of Medical and Surgical Sciences-DIMEC,

S.Orsola-Malpighi Hospital, Alma Mater StudiorumUniversity of Bologna, Bologna, Italy

\author{
Address for correspondence: \\ Vito Sansone \\ Unit of Internal Medicine, \\ University of Bologna, \\ Azienda Ospedaliero \\ Universitaria S.Orsola- \\ Malpighi, \\ Bologna, Italy \\ vito.sansone@studio.unibo.it
}

Received: 18.11.2019

Accepted: 17.04.2020

\begin{abstract}
Multiple focal liver lesions were incidentally detected in a patient screened by ultrasound for a recent diagnosis of lower limb deep vein thrombosis, for which anticoagulation had been initiated. Past medical history reported a post-traumatic splenectomy 15 years before. Magnetic resonance imaging (MRI) and contrast-enhanced ultrasound (CEUS) showed a subcapsular lesion in liver segment 5 consistent with focal nodular hyperplasia (FNH) and multiple other nodules, with a different pattern from the former, judged as probable hepatic adenomas by MRI but probable hemangiomas by CEUS (hyperenhancement in the late phase). Therefore, another MRI with gadoxetic acid was performed. The diagnosis of FNH was confirmed. The other lesions showed an hyperenhancing pattern in the arterial phase with progressive wash-out in the portal and late phase and marked hypointensity in the hepatobiliary phase. This pattern apparently confirmed the hypothesis of adenomas, with a potential risk of malignancy due to the hepatobiliary phase pattern and the recent occurrence of deep vein thrombosis. Due to the inherent risk of spontaneous bleeding from subcapsular adenomas increased by the ongoing anticoagulant therapy and the recommendation of international guidelines to resect adenomas in male subjects, the patient was directly offered surgery. Pathology of the resected specimens confirmed one FNH but demonstrated intrahepatic splenosis for all other lesions. This case suggests that in the setting of previous splenic trauma any discrepancy between MRI and CEUS findings should lead one to consider also the hypothesis of intrahepatic splenosis. .
\end{abstract}

Key words: ultrasound- magnetic resonance imaging - splenosis - primovist - gadoxetic acid.

Abbreviations: CEUS: contrast-enhanced ultrasound; FNH: focal nodular hyperplasia; LMWH: low molecular weight heparin; MRI: magnetic resonance imaging; US: ultrasound.

\section{INTRODUCTION}

Splenosis is a rare condition defined as the autotransplantation of ectopic splenic tissue in other anatomic compartments, first described in 1939 [1]. It is a benign condition and can be either congenital (accessory spleen) or acquired, mainly after a history of splenic trauma (with an occurrence in 26-67\% of patients [2]) or a splenectomy [3]. Splenic implants are usually multiple, scattered throughout the whole peritoneal cavity, and in different parenchymatous organs [4-6]. Uncommon sites of implantation have been described, including the thoracic cavity and the brain. Intrahepatic splenosis is defined as the autoimplantation of ectopic splenic tissue in the liver parenchyma. There are two hypotheses on the mechanisms of splenic tissue spread. The first hypothesis postulates a "seeding" of the splenic pulp after the trauma. Alternatively, hepatic splenosis could result from a hematogenous spread of the splenic cells through the splenic vein. Hepatic splenosis is a rare condition and its diagnosis is challenging with non-invasive means, as it lacks specific radiologic features, mimicking other hepatic masses, such as adenoma or hepatocellular carcinoma [7].

We present the case of a patient with intrahepatic splenosis provoked by a trauma, diagnosed after a surgical intervention for suggestive adenomatosis on imaging procedures. We discuss in detail the diagnostic process with highlights on Contrast-Enhanced Ultrasound (CEUS) and Magnetic Resonance Imaging (MRI) features. 


\section{CASE REPORT}

A 46-year-old man was referred to our institution for the incidental finding, during an abdominal ultrasound (US) of multiple focal liver lesions, not previously known. The most prominent ones were in segments $>5(49$ x $45 \mathrm{~mm}), 4 \mathrm{a}(29 \mathrm{x}$ $22 \mathrm{~mm}), 6(12 \times 23 \mathrm{~mm})$ and $8(14 \times 8 \mathrm{~mm})$ (Fig. 1). Medical history included: splenectomy following a road accident 15 years before, Factor V Leiden heterozygosis, and deep vein thrombosis that had occurred 2 months before, for which low molecular weight heparin (LMWH) was still ongoing. He was in good clinical conditions and his general blood tests were normal.

Shortly after detection of the multiple focal liver lesions and before referral to our center, the patient had already undergone upper abdominal MRI with standard gadolinium-based contrast agent. This imaging technique showed a subcapsular lesion in hepatic segment 5 with hyperenhancement in the arterial phase persisting throughout the venous phase, consistent with focal nodular hyperplasia (FNH). Additionally, 6 other liver nodules were detected, but with a different contrast pattern from the previous, consistent with probable adenomas, showing hyperenhancement in the arterial phase and washout in portal and late phases, and hypoenhancement in the hepatobiliary phase. CEUS was performed and the vascular pattern was suggestive for one FNH (Fig. 2) and multiple hemangiomas (Fig. 3) due to the persistent hyperenhancement in the portal and late phases.

To verify the discrepancy between MRI and CEUS, suggesting two different diagnoses (adenoma vs hemangioma), we decided to repeat MRI at our institution, but using a hepatocyte-specific contrast agent (gadoxetic acid, Gd-EOBDTPA, Primovist ${ }^{\oplus}$ ). The lesion in segment 5 was confirmed consistent with a FNH of $43 \times 36 \mathrm{~mm}$, while at least 12 other lesions were identified in all liver segments, the largest of $25 \times 19 \mathrm{~mm}$ in segment $4 \mathrm{a}$, all showing hypointensity (or "enhancement defect") in the post vascular hepatocyte-specific phase (Fig. 4).

These 12 lesions presented hyperenhancement in the arterial phase with progressive wash-out in the portal and late phase and marked hypointensity in the hepatobiliary phase. The pattern, especially in the hepatobiliary phase, differed from the typical FNH, which appeared instead hyperenhanced in the hepatobiliary phase (Fig. 5). The imaging pattern of the multiple lesions was suggestive for adenomatosis which was potentially worrisome (for the theoretical risk of malignant transformation of adenomas) considering the strong enhancement defect in the hepatobiliary phase and the male gender.

A few small masses were reported in the left upper abdominal quadrant and were ascribed to intraabdominal residual splenic tissue (Fig. 6).

Given the ongoing long-term anticoagulant therapy and the consequent risk of intraperitoneal bleeding from the presumed multifocal adenomatosis and - to a much lesser extent - from the subcapsular FNH, since partially abutting from the liver profile and given the known risk of malignant transformation of adenomas in males, the patient was offered surgery. Resection had in fact, on one hand the objective to avoid the bleeding risk, especially in the setting of long-term warfarin therapy; on the other hand, this approach had also the objective to verify the type of the presumed adenomas.

Surgery consisted of wedge resection of the lesion in segment $5(\mathrm{FNH})$ and of the largest additional nodules. Pathology of the resected specimens confirmed one FNH but demonstrated intrahepatic splenosis for the other lesions with no finding of any adenoma. Twelve months after surgery the patient was alive and in a good health condition, without new focal liver lesions.

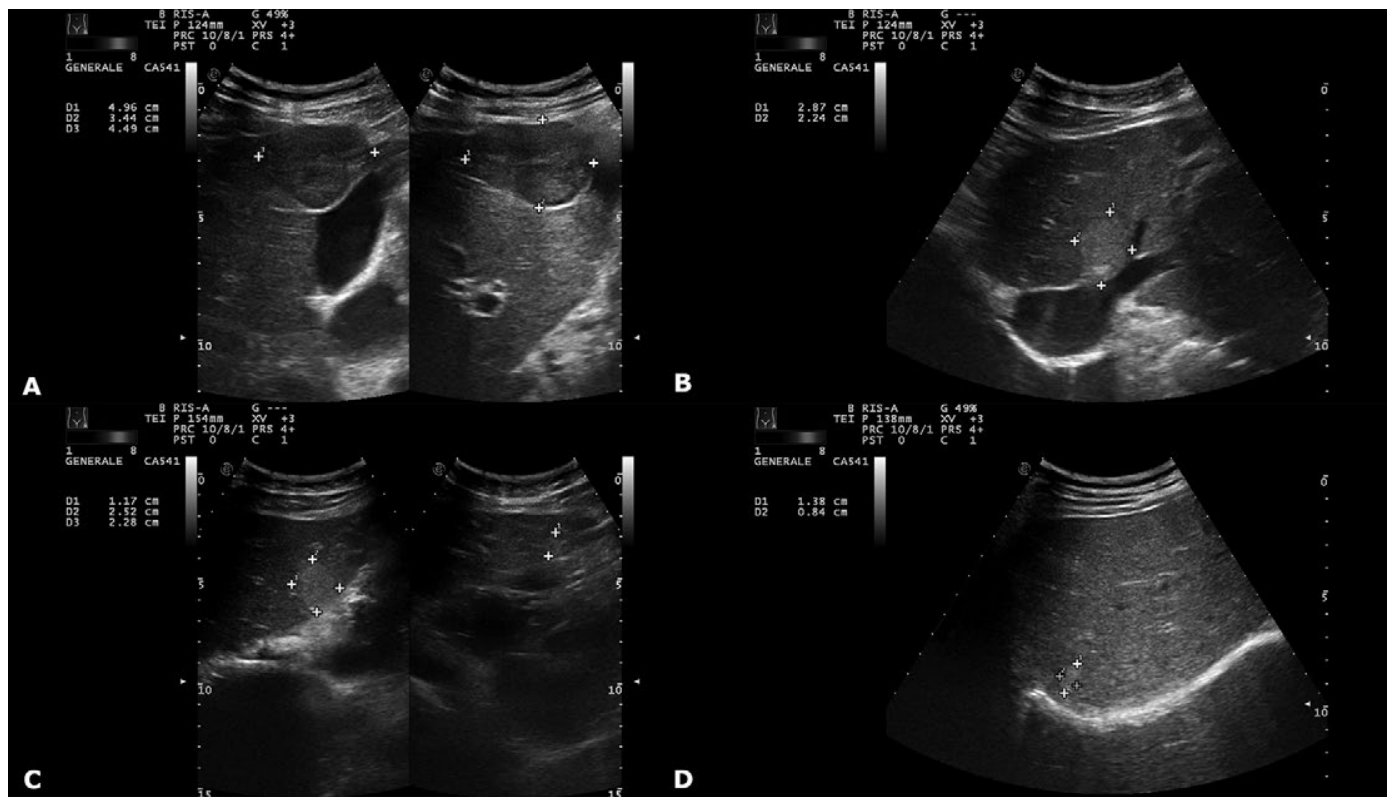

Fig. 1. Ultrasound appearance of the focal liver lesions: A - focal nodular hyperplasia, segment 5, 49 x $45 \mathrm{~mm}$; B - hepatic splenosis in segment 4a, measuring $29 \times 22 \mathrm{~mm}$; C - another lesion of hepatic splenosis in segment $6,12 \times 23 \mathrm{~mm}$ and, D- in segment $8,14 \times 8 \mathrm{~mm}$. 


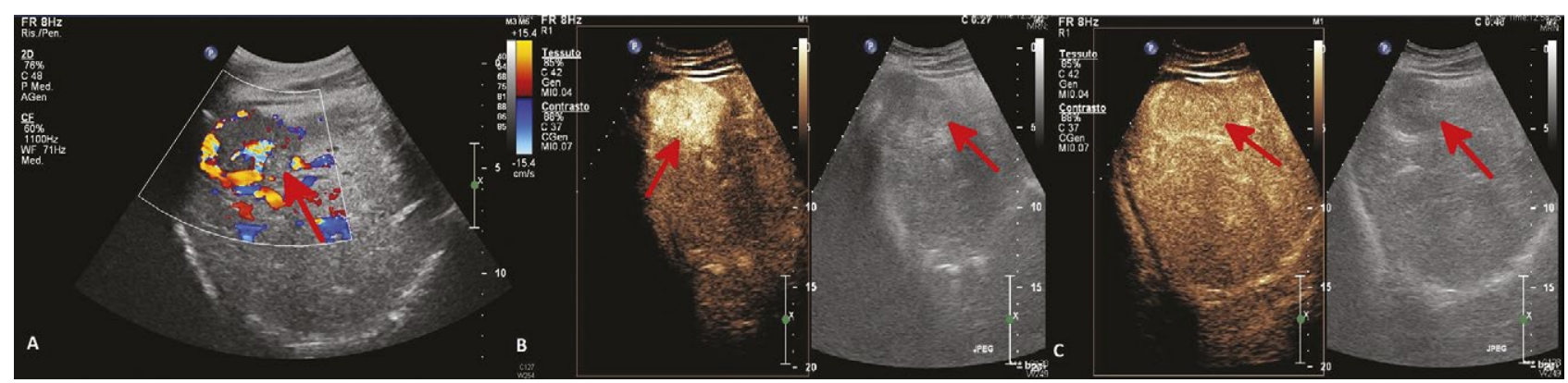

Fig. 2. US Doppler and CEUS of the focal nodular hyperplasia. A - Color Doppler shows a central feeding artery and partially evident arterial radial distribution; B - At CEUS the lesion shows hyperenhancement in the arterial phase; C - isoenhancement in the portal phase. The overall pattern is consistent with focal nodular hyperplasia.

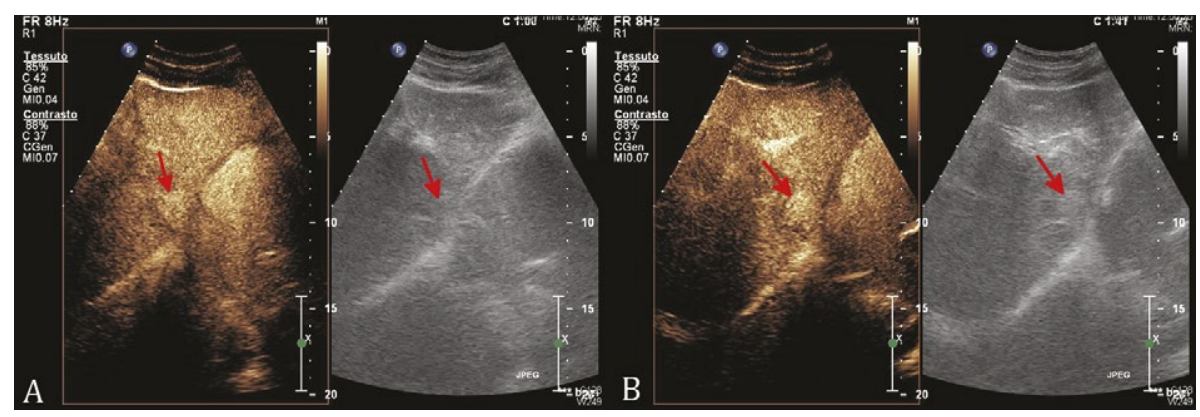

Fig. 3. CEUS pattern of one intrahepatic splenosis, located subcapsular and posterior in the left lobe. The lesion appeared hyperenhancing in the arterial phase (not shown) and remained hyperenhancing in the early (A) (1m after the injection) and late (B) portal phases ( $1 \mathrm{~m} 41 \mathrm{~s}$ after injection). The CEUS pattern was considered suggestive of shunt hemangioma, as the conventional B-mode appearance was as well.

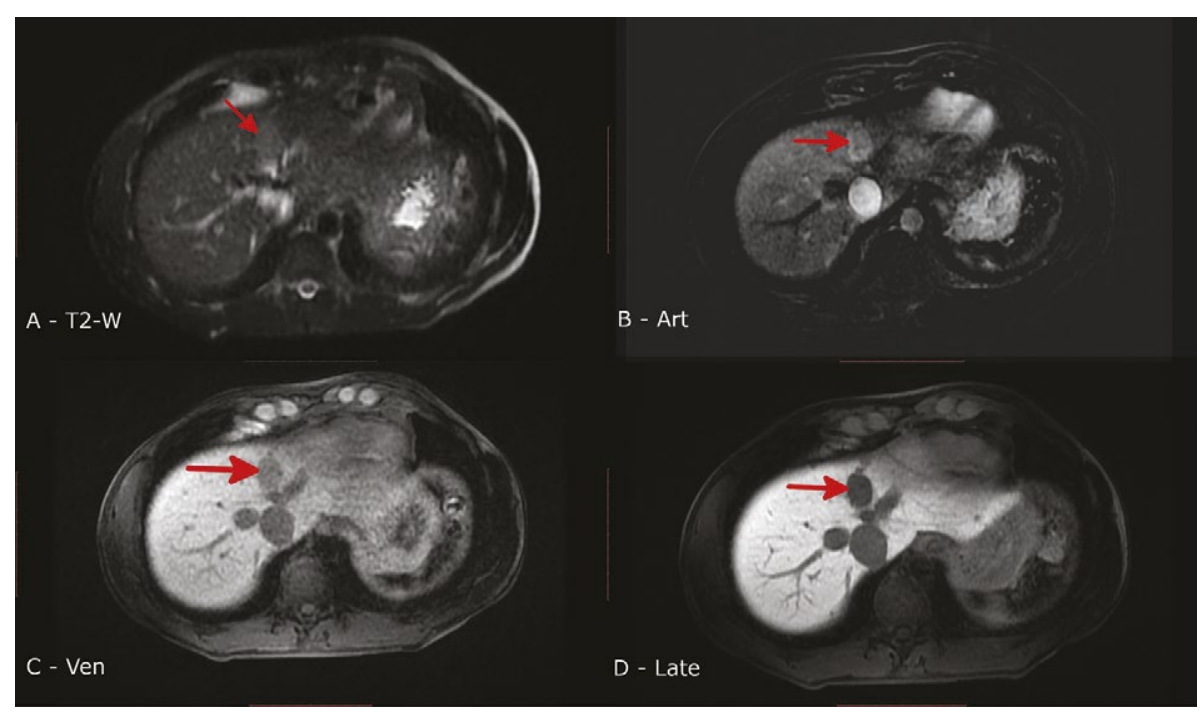

Fig. 4. MRI of the largest lesion of intrahepatic splenosis, located in segment 4 . The lesion is weakly hyperdense in the T2 weighted phase (A). After contrast injection, the lesion shows hyperenhancement in the arterial phase (B), washout in the venous (C) and an enhancement defect in the hepatobiliary phase (D).

\section{DISCUSSION}

The lack of the correct diagnosis of intrahepatic splenosis and the apparent discrepancy among the various imaging techniques deserve some considerations.

Gadoxetic acid is a contrast agent with a hepatobiliary secretive phase. It gained attention in the clinical setting as an adjunctive tool in the diagnosis of focal liver lesions: hypointensity in the hepatobiliary phase is indicative of different entities, which in the very vast majority of cases correspond to: 1) non-secretory (hence, malfunctioning) hepatocytes [8], such as in the case of hepatocellular carcinoma [9] or of some adenomas, 2) non-hepatocellular cells (e.g. other primary or metastatic liver tumours), 3) fluid containing spaces 


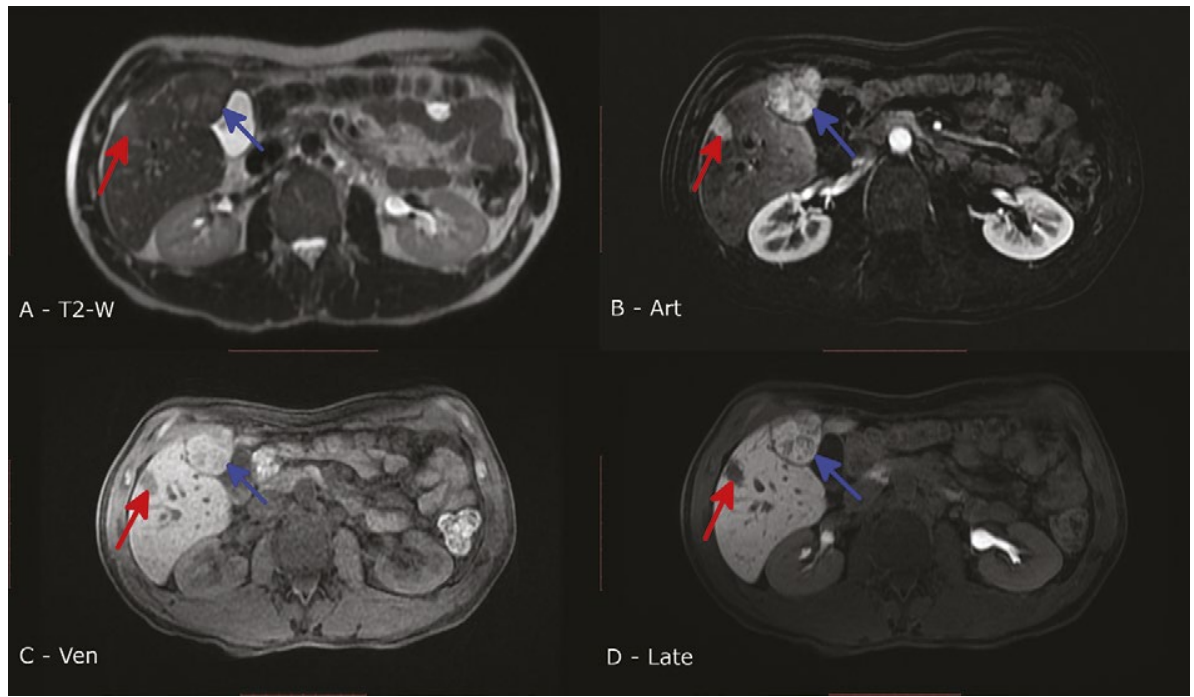

Fig. 5. MRI: T2 weighted phase (A) and contrast patterns (arterial phase $B$, venous phase $C$ and hepatobiliary phase D) of: - focal nodular hyperplasia in segment 5 , pointed out by the blue arrows, and of a subcapsular splenosis, pointed out by the red arrows. Focal nodular hyperplasia and intrahepatic splenosis differ markedly mainly in the venous and hepatobiliary phases.

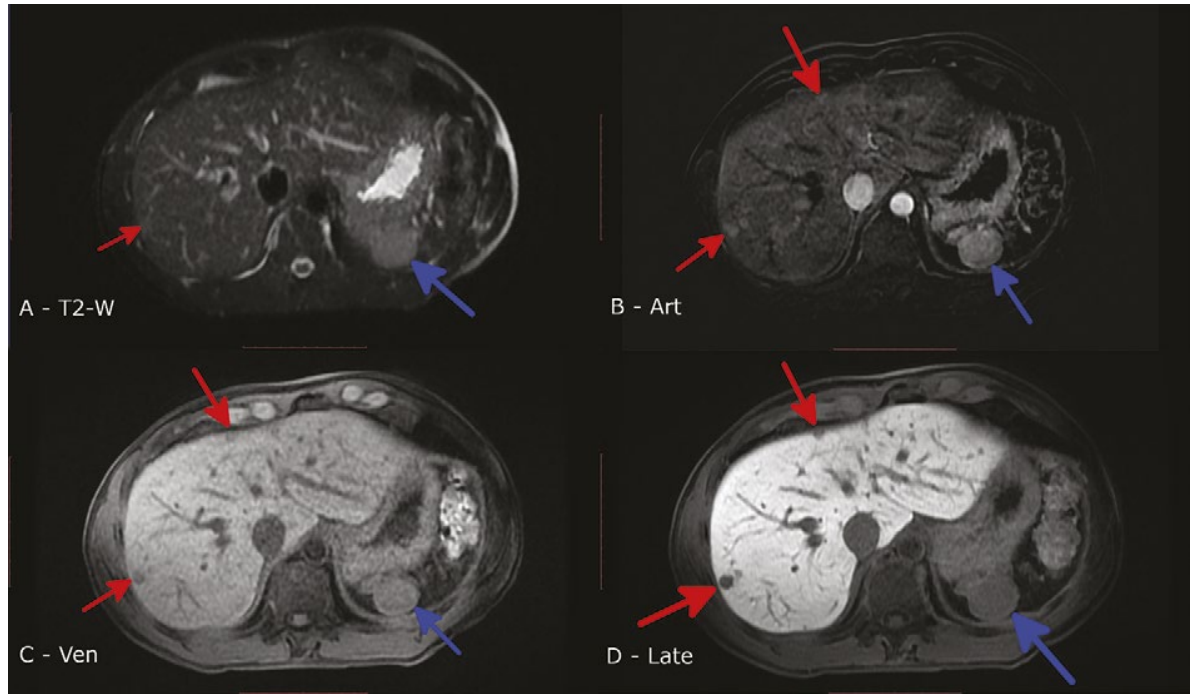

Fig. 6. MRI of abdominal splenosis, located in the left upper abdomen (blue arrows). If the liver is held as a comparator, the masses show indeed the same patterns as the intrahepatic splenosis (red arrows). They are weakly hyperdense in the T2 weighted phase (A), show hyperenhancement in the arterial phase (B), washout in the venous (C) and an enhancement defect in the hepatobiliary phase (D). However, being located far from the liver, the latter was not expected to be held as a comparator at the time of the MRI scanning.

(e.g. hemangioma, cysts, fluid collections, etc). In the current case, however, the enhancement defect in the hepatocytespecific phase corresponded to an ectopic, splenic tissue, an unsuspected finding, extremely rare: an almost exceptional benign solid liver lesion of non-hepatocellular nature with enhancement defect in the hepatobiliary phase. Indeed, the histological finding is consistent with the absence of hepatocytes, justifying the enhancement defect. The alternative possibility of "hypervascular" or "shunt" hemangioma was ruled out by the lack of hyperintensity in T2.

The diagnosis was oriented to presumed adenomas based on arterial phase hyperenhancement and multifocality (with or without malignant transformation, as adenomas progressed to hepatocellular carcinoma could have been an alternative possibility, hypothetically favoring the onset of deep vein thrombosis). Beta-catenin adenomas account for the 10-20\% of total adenomas, and their identification is of the utmost importance, as they harbor an increased risk of malignant transformation [10].

Molecular classification of adenomas has an impact on prognosis [11] and therapeutic decisions [12]. Beta-catenin mutated adenomas have an increased risk of malignant transformation, whilst sonic hedgehog positive adenomas are particularly prone to symptomatic bleedings, especially if large in size [11]. Therefore, these two conditions benefit most from surgery.

In the case of the absence of malignant cells at the standard pathological examination, beta-catenin mutation 
should be evaluated, as its presence implies a certain risk of a subsequent malignant transformation [10, 13]. The hypothesis of malignancy could not be discarded "a priori", since theoretically the presence of malignancy could also have been a factor favoring the onset of deep vein thrombosis and would impact the overall treatment strategy including referral for transplantation. The possibility to obtain histology and molecular type of the presumed adenoma by percutaneous biopsy was held at an excessive risk of complications, given the small size and peripheral/subcapsular location of the potential target lesions and their nature.

Liver biopsy is mostly regarded as a safe procedure in general, nonetheless, relevant complications such as seeding, or bleeding have to be considered. Seeding may occur in the biopsy of any malignancy, but specific rates of seeding in malignant adenomas have not been described given its relative rarity, so we can only refer to seeding risk in hepatocellular carcinoma in general (up to 7\%) [14]. Clinically severe hemorrhage after ultrasound guided biopsy of the liver has been reported at rates of $0.2 \%$ in a recent series [15] and of $0.3-0.5 \%$ in an older series [16]. In a series of cases of 61 biopsies for presumed hepatic adenoma, only one single case of significant major bleeding was reported, corresponding to approximately $2 \%$ of the final population [17]. Moreover, a fine needle biopsy in adenomas was held to be not enough to rule out malignancy in adenomas in a male patient, for whom guidelines would recommend resection anyway [10]. In fact, malignant transformation may take place focally in the early stages and biopsy may often provide false negative results for cancer [17].

Unfortunately, no imaging feature is able to identify betacatenin mutated adenomas. Only steatotic adenomas [10], which do not suffer from beta-catenin mutations, can be recognized with MRI [10]. Histological evaluation is instead advised for all adenomas which remain at risk to carry the betacatenin mutation and this can be obtained by biopsy sampling. However, a biopsy suffers a risk of significant bleeding in adenoma (approximately $2 \%$ ), but these rates are expected to be much higher when lesions are superficially located. A biopsy was not performed in our case, not only due to the subcapsular position of the lesions associated with an increased risk of bleeding, but rather because assuming an instance of adenomas, one fine needle biopsy would not have spared the patient from surgery. The reasons were the multiplicity of the lesions and the recommendation to resect adenomas in males, since they tend to suffer much more commonly from betacatenin mutated adenomas [10].

Focal nodular hyperplasia is a benign focal liver lesion that in most cases is managed conservatively: the usual indications for surgery are the presence of symptoms such as abdominal discomfort or jaundice. [18] The surgical resection of the FNH in this case has therefore to be considered an exception, related to the fact that laparotomy had already been indicated because of the presumed adenomas and the fact that the patient had to receive anticoagulation, which lead us to speculate that a large subcapsular abutting liver lesion, could be at a higher risk of bleeding.

In this specific case, more weight should have been given to the past medical history of splenic rupture to raise the possibility of intrahepatic splenosis among other alternatives, even though hardly any non-invasive diagnostic tool could have safely avoided surgery. It is worthy of remarking for future similar situations that adenomas tend not to be hyperenhancing in the late phase at CEUS, a condition which indicates a benign nature, but is typical of hemangiomas and some FNHs. Intrabdominal splenosis is an uncommon diagnosis although it has been reported to occur in the liver [4], as well as in the pancreas [5] and other abdominal organs [6]. We conducted a PubMed search for hepatic splenosis cases with the string: splenosis AND liver OR „hepatic splenosis” and found a total of 50 cases with either CEUS ( 2 cases) or MRI (49) images described in 43 articles (Supplementary Table I), confirming the rarity of this condition. This is a condition which can be easily overlooked by imaging operators and is therefore worth bringing attention to. Intrahepatic splenosis at MRI appears as a well-defined lesion hypointense in T1-w sequences and hyperintense in T2-w sequences, with a hypointense capsule in both sequences $[19,20]$. Diffusion-weighted imaging sequences show a high intensity signal and a corresponding low one on apparent diffusion coefficient maps [19].

After contrast injection, a homogeneous uptake is reported in the arterial phase, which becomes heterogeneous in the venous phase and is lower than surrounding liver parenchyma in the delayed phase $[20,21]$. The CEUS pattern of hepatic splenosis includes variable arterial phase enhancement and sustained enhancement throughout the portal and sinusoidal phases, but the total number of reported cases (three including the present one), prevents any generalization of typical patterns [22, 23].

\section{CONCLUSIONS}

Liver lesions in the presence of consistent medical history (previous splenic trauma or rupture), enhancement defect in the hepatobiliary phase following injection of hepatocytespecific MRI contrast agents, late hyperenhancement at CEUS in the absence of hemangiomas at T2 weighted MRI should raise suspicion of hepatic splenosis. We cannot draw instead any conclusion regarding the B-mode ultrasound appearance of hepatic splenosis, which in this case resembled hemangiomas, based on one single case.

\section{Conflicts of interest: None to declare.}

Authors' contributions: V.S. drafted the manuscript. L.F. performed the CEUS examination. R.G. interpreted the MRI. F.T., M.C. and F.P. critically revised the manuscript. All authors critically revised the manuscript, approved the final version to be published, and agree to be accountable for all aspects of the work.

Supplementary material: To access the supplementary material visit the online version of the J Gastrointestin Liver Dis at http://dx.doi. org/10.15403/jgld-617

\section{REFERENCES}

1. Buchbinder JH, Lipkoff CJ. Splenosis: Multiple peritoneal splenic implants following abdominal injury: A report of a case and review of the literature. Surgery 1939;6:927-934. 
2. Wu C, Zhang B, Chen L, Zhang B, Chen X. Solitary perihepatic splenosis mimicking liver lesion: a case report and literature review. Medicine (Baltimore) 2015;94:e586. doi: 10.1097/MD.0000000000000586

3. Depypere L, Goethals M, Janssen A, Olivier F. Traumatic rupture of splenic tissue 13 years after splenectomy. A case report. Acta Chir Belg 2009;109:523-526. doi:10.1080/00015458.2009.11680476

4. Leong CW, Menon T, Rao S. Post-traumatic intrahepatic splenosis mimicking a neuroendocrine tumour. BMJ Case Rep 2013;2013:bcr2012007885. doi:10.1136/bcr-2012-007885

5. Herédia V, Altun E, Bilaj F, Ramalho M, Hyslop BW, Semelka RC. Gadolinium- and superparamagnetic-iron-oxide-enhanced MR findings of intrapancreatic accessory spleen in five patients. Magn Reson Imaging 2008;26:1273-1278. doi:10.1016/j.mri.2008.02.008

6. Sorensen SF, Mortensen FV, Hellberg Y, Ladekarl M. Diffuse abdominal splenosis mimicking peritoneal metastases in a 35-year-old man with a resectable carcinoma of the ampulla of vater. Case Rep Oncol 2013;6:467-471. doi:10.1159/000355233

7. Wang WC, Li XF, Yan ZL, et al. Intrahepatic splenosis mimics hepatocellular carcinoma in a patient with chronic hepatitis B. Medicine (Baltimore) 2017;96:e8680. doi:10.1097/MD.0000000000008680

8. Van Beers BE, Pastor CM, Hussain HK. Primovist, eovist: What to expect? J Hepatol 2012;57:421-429. doi:10.1016/j.jhep.2012.01.031

9. Duncan JK, Ma N, Vreugdenburg TD, Cameron AL, Maddern G. Gadoxetic acid-enhanced MRI for the characterization of hepatocellular carcinoma: A systematic review and meta-analysis. J Magn Reson Imaging 2017;45:281-290. doi:10.1002/jmri.25345

10. European Association for the Study of the Liver (EASL). EASL Clinical Practice Guidelines on the management of benign liver tumours. J Hepatol 2016;65:386-398. doi:10.1016/j.jhep.2016.04.001

11. Nault J, Paradis V, Cherqui D, Vilgrain V, Zucman-Rossi J. Molecular classification of hepatocellular adenoma in clinical practice. J Hepatol 2017;67:1074-1083. doi:10.1016/j.jhep.2017.07.009

12. Bioulac-Sage P, Cubel G, Taouji S, et al. Immunohistochemical Markers on Needle Biopsies Are Helpful for the Diagnosis of Focal Nodular Hyperplasia and Hepatocellular Adenoma Subtypes. Am J Surg Pathol 2012;36:1691-1699. doi:10.1097/PAS.0b013e3182653ece

13. Zucman-Rossi J, Jeannot E, Nhieu JT, et al. Genotype-phenotype correlation in hepatocellular adenoma: New classification and relationship with HCC. Hepatology 2006;43:515-524. doi:10.1002/ hep. 21068

14. SparchezZ, Mocan T. Contemporary role of liver biopsy in hepatocellular carcinoma. World J Hepatol 2018;27;10:452-461. doi:10.4254/wjh.v10. i7. 452

15. Strobel D, Bernatik T, Blank W, et al. Incidence of Bleeding in 8172 Percutaneous Ultrasound-Guided Intraabdominal Diagnostic and Therapeutic Interventions - Results of the Prospective Multicenter DEGUM Interventional Ultrasound Study (PIUS Study). Ultraschall Med 2015;36:122-131. doi:10.1055/s-0034-1399282

16. Grant A, Neuberger J. Guidelines on the use of liver biopsy in clinical practice. British Society of Gastroenterology. Gut 1999;45(Suppl 4):IV1IV11. doi:10.1136/gut.45.2008.iv1

17. Doolittle DA, Atwell TD, Sanchez W, et al. Safety and outcomes of percutaneous biopsy of 61 hepatic adenomas. AJR Am J Roentgenol 2016;206:871-876. doi:10.2214/AJR.15.15301

18. Perrakis A, Vassos N, Grützmann R, Croner RS. What is changing in indications and treatment of focal nodular hyperplasia of the liver. Is there any place for surgery? Ann Hepatol 2017;16:333-341. doi:10.5604/16652681.1235475

19. Gandhi D, Sharma P, Garg G, Songmen S, Solanki S, Singh T. Intrahepatic splenosis demonstrated by diffusion weighted MRI with histologic confirmation. Radiol Case Reports 2020;15:602-606. doi:10.1016/j.radcr.2020.02.022

20. Lin WC, Lee RC, Chiang JH, et al. MR Features of Abdominal Splenosis. AJR Am J Roentgenol 2003;180:493-496. doi:10.2214/ ajr.180.2.1800493

21. Somsap K, Chamadol N, Titapun A, Pairojkul C, Sangkhamanon S. MR imaging findings of a patient with isolated intrahepatic splenosis mistaken for hepatocellular carcinoma. BJR Case Rep 2016;3:20150242. doi:10.1259/bjrcr.20150242

22. Ferraioli G, Di Sarno A, Coppola C, Giorgio A. Contrast-Enhanced LowMechanical-Index Ultrasonography in Hepatic Splenosis. J Ultrasound Med 2006;25:133-136. doi:10.7863/jum.2006.25.1.133

23. Grambow E, Weinrich M, Zimpfer A, Kloker K, Klar E. Ectopic Spleen Tissue - an Underestimated Differential Diagnosis of a Hypervascularised Liver Tumour. Viszeralmedizin 2015;31:445-447. doi:10.1159/000442115 